\title{
Numeric and Analytic Investigation on Phase Diagrams and Phasetransitions of the $v=2 / 3$ Bilayer Fractional Quantum Hall Systems
}

\author{
Y. D. Zheng, T. Sorita \\ Research and Development Department, Mitsubishi Electric (China) Company Limited, Shanghai, China \\ Email: zhengyd09@163.com
}

How to cite this paper: Zheng, Y.D. and Sorita, T. (2018) Numeric and Analytic Investigation on Phase Diagrams and Phasetransitions of the $v=2 / 3$ Bilayer Fractional Quantum Hall Systems. Journal of Applied Mathematics and Physics, 6, 667-676. https://doi.org/10.4236/jamp.2018.64060

Received: March 19, 2018

Accepted: April 9, 2018

Published: April 12, 2018

\begin{abstract}
The phase diagrams and phase transitions of a typical bilayer fractional quantum Hall $(\mathrm{QH})$ system with filling factor $v=2 / 3$ at the layer balanced point are investigated theoretically by finite size exact-diagonalization calculations and an exactly solvable model. We find some basic features essentially different from the bilayer integer $\mathrm{QH}$ systems at $v=2$, reflecting the special characteristics of the fractional QH systems. The degeneracy of the ground states occurs depending on the difference between intralayer and interlayer Coulomb energies, when interlayer tunneling energy $\left(\Delta_{\mathrm{SAS}}\right)$ gets close to zero. The continuous transitions of the finite size systems between the spin-polarized and spin-unpolarized phases are determined by the competition between the Zeeman energy $\left(\Delta_{\mathrm{Z}}\right)$ and the electron Coulomb energy, and are almost not affected by $\Delta_{\mathrm{SAS}}$.
\end{abstract}

\section{Keywords}

Bilayer Fractional Quantum Hall Systems, Phase Diagrams, Phase Transition, Exactdiagonalization, Exactly Solvable Model

\section{Introduction}

Quantum Hall (QH) effect [1] [2] [3] [4] [5], which rivals superconductivity in its fundamental significance, has attracted a great deal of experimental and theoretical interest since its discovering. Especially, fractional QH (FQH) systems exhibit a variety of many body quantum phenomena, due to the complete domination of the electron Coulomb interactions. In a more complicated case, the bilayer QH systems with both spin and layer (pseudospin) degrees of freedom, four sub energy levels are formed in the lowest Landau level (LLL), and the 
ground states are to be determined by various factors such as interlayer/intralayer Coulomb energies $\left(\Delta_{\mathrm{C}}\right)$, Zeeman energy $\left(\Delta_{\mathrm{Z}}\right)$, interlayer tunneling energy $\left(\Delta_{\mathrm{SAS}}\right)$ and bias energy $\left(\Delta_{\text {bias }}\right)$, etc. One expects reasonably that there exist rich quantum phases and many novel properties in the systems [6] [7].

The bilayer QH systems with filling factor $v=2 / m$ should be of the same type for any odd integer $\mathrm{m}$. In this type of systems, the spin and pseudospin indices compete with each other, and the ground states are quite nontrivial because there are several ways to fill electrons into two sub energy levels in the LLL. Up to now, most of theoretical studies have focused on the $v=2$ bilayer integer $\mathrm{QH}$ (IQH) systems based on Hartree-Fock analysis [8] [9] [10] [11] [12] [13], effective bosonic spin theory [14] [15], exact-diagonalization calculations [16] as well as effective quantum field theory, and have identified three phases, the ferromagnetic (FM), the symmetric (SYM) and the canted antiferromagnetic (CAF) phases in the ground states. On the other hand, the $v=2 / 3$ bilayer $\mathrm{QH}$ system is a typical bilayer FQH system of this type, and can be regard as a best example of strongly correlated two-dimensional electron system for investigating the interplay of those entangled energy factors indicated above. However, even the basic problems, the ground states and the basic phase diagram of the $v=2 / 3$ system are still leaved uninvestigated from the theoretical viewpoint. Because of the existence of the degeneracy in the ground state as indicated below, in many cases, the $v=2 / 3$ bilayer $\mathrm{QH}$ system cannot simply be mapped to the $v=2$ bilayer $\mathrm{QH}$ system based on the composite-fermion picture, and must be investigated directly by the microscopic theories and the numerical calculations.

Motivated by the present situation mentioned above, as the first step, in this paper, we employ the numerical and traditional analytic methods to investigate the finite size bilayer FQH systems. We report on some basic features of the ground states in the $v=2 / 3$ bilayer $\mathrm{QH}$ systems at the layer balanced point $\left(\Delta_{\text {bias }}\right.$ $=0$ ) and provide evidential quantitative results from exact-diagonalization (ED) [16] [17] [18] [19] [20] numerical calculations and analytic approaches carried out at $v=2 / 3$. These features are essentially different from those in the $v=2$ systems, and reflect the special characteristics of the bilayer FQH systems. 1) At $v=2 / 3$, because the number of electrons is less than that of Landau sites in one sub energy level, in the small $\Delta_{\mathrm{SAS}}$ limit, the degeneracy of the ground states occurs depending on the relative strength of the intralayer and interlayer Coulomb energies. Contrarily, at $v=2$, because two sub energy levels in the LLL are filled by electrons, the ground states are non-degenerate even if $\Delta_{\mathrm{SAS}}$ vanishes. 2) At least, the spin-polarize (SP) and spin-unpolarized (SU) phases exist in the $v=$ $2 / 3$ bilayer $\mathrm{QH}$ systems. The phase transitions between them are continuous in the finite size systems, and are determined by the competition between $\Delta_{\mathrm{Z}}$ and Coulomb energy $\Delta_{\mathrm{C}}$, not that between $\Delta_{\mathrm{Z}}$ and $\Delta_{\mathrm{SAS}}$, as in the $v=2$ systems. The experimental results so far seem to support the conclusions above.

\section{Exact-Diagonalization Method}

We choose a finite size system with rectangular geometry for ED calculations. 
Periodic boundary conditions are imposed on the rectangular cell of area $a \times b$ along the $\mathrm{x}$ and $\mathrm{y}$ axes, with the periodicities $a$ and $b$, respectively. For simplicity, Landau level mixing and finite thickness of the system are not considered. Within the LLL, the Hamiltonian at the layer balanced point is described by Equations (1) and (2) as follows:

$$
\begin{gathered}
\hat{H}=\hat{H}_{\mathrm{t}}+\hat{H}_{\mathrm{z}}+\hat{H}_{\mathrm{int}}, \\
\hat{H}_{\mathrm{t}}=-\frac{\Delta_{\mathrm{SAS}}}{2} \sum_{j \sigma}\left(\hat{c}_{j f \sigma}^{+} \hat{c}_{j b \sigma}+\hat{c}_{j b \sigma}^{+} \hat{c}_{j f \sigma}\right), \\
\hat{H}_{\mathrm{Z}}=-\frac{\Delta_{\mathrm{Z}}}{2} \sum_{j \mu}\left(\hat{c}_{j \mu \uparrow}^{+} \hat{c}_{j \mu \uparrow}-\hat{c}_{j \mu \downarrow}^{+} \hat{c}_{j \mu \downarrow}\right), \\
\hat{H}_{\mathrm{int}}=\frac{1}{2} \sum_{j_{1}-j_{4}} \sum_{\mu_{1}-\mu_{4} \sigma_{1} \sigma_{2}} V_{j_{1} j_{2} j_{3} j_{4}}\left(F_{\mu_{1} \mu_{2} \mu_{3} \mu_{4}}\right) \times \hat{c}_{j_{1} \mu_{1} \sigma_{1}}^{+} \hat{c}_{j_{2} \mu_{2} \sigma_{2}}^{+} \hat{c}_{j_{3} \mu_{3} \sigma_{2} \sigma_{2}} \hat{c}_{j_{4} \mu_{4} \sigma_{1}}, \quad(1) \\
\frac{V_{j_{1} j_{2} j_{3} j_{4}}\left(F_{\mu_{1} \mu_{2} \mu_{3} \mu_{4}}\right)=}{4 \pi \varepsilon} \frac{1}{2 a b} \delta^{\prime}\left(j_{1}+j_{2}, j_{3}+j_{4}\right) \sum_{s(q \neq 0)} e^{-i 2 \pi\left(\frac{s\left(j_{1}-j_{3}\right)}{M}\right.}\left[\frac{2 \pi}{q} e^{-q^{2} I_{B}^{2} / 2} F_{\mu_{1} \mu_{2} \mu_{3} \mu_{4}}\right]_{q_{x}=\frac{2 \pi}{a} s, q_{y}=\frac{2 \pi}{b}\left(j_{4}-j_{1}\right)}, \\
F_{\text {ffff }}=F_{b b b b}=1, F_{f b b f}=F_{b f f b}=e^{-q d}, \text { otherwise } F_{\mu_{1} \mu_{2} \mu_{3} \mu_{3} \mu_{4}}=0,
\end{gathered}
$$

where $\hat{H}_{\mathrm{t}}, \hat{H}_{\mathrm{z}}$ and $\hat{H}_{\mathrm{int}}$ represent the interlayer tunneling energy, Zeeman energy and two-body Coulomb interaction energy terms, respectively. $\hat{c}_{j \mu \sigma}^{+}$ $\left(\hat{c}_{j \mu \sigma}\right)$ denotes electron creation (annihilation) operator. $j>0$ is the y-direction momentum (Landau site) index (y-direction momentum $j_{\mathrm{y}}=j-1$ ), $\mu=\mathrm{f}, \mathrm{b}$ is the layer index that labels the front and back layers, and the spin denotation $\sigma=$ $\uparrow, \downarrow$ represents the up and down spins. The coefficients in the $\hat{H}_{\text {int }}$ term are given by Equation (2) [17] [18], where $I_{\mathrm{B}}$ means the magnetic length, $d$ is the layer separation, $q_{\mathrm{x}}\left(q_{\mathrm{y}}\right)$ indicates the single-electron wave numbers in $\mathrm{x}(\mathrm{y})$ direction. We define $M$ as the degeneracy of the single Landau level. Only when $j_{1}=j_{2}$ $(\bmod M), \delta^{\prime}\left(j 1, j_{2}\right)=1$, otherwise, $\delta^{\prime}\left(j_{1}, j_{2}\right)=0$.

The total number of electrons in the system is defined by $N_{\mathrm{e}}$. The $N_{\mathrm{e}}$-electron basis vector is expressed by $\varphi_{r}=\hat{c}_{j_{1} \mu_{1} \sigma_{1}}^{+} \hat{c}_{j_{2} \mu_{2} \sigma_{2}}^{+} \ldots \hat{c}_{j_{\mathrm{Ne}} \mu_{\mathrm{Ne}} \sigma_{\mathrm{Ne}}}^{+}|0\rangle$ (e.g., $\{2 \mathrm{f} \uparrow, 3 \mathrm{~b} \uparrow, 5 \mathrm{f}$ $\downarrow, 6 \mathrm{~b} \downarrow\}=\hat{\mathbf{c}}_{2 \mathrm{f} \uparrow}^{+} \hat{\mathbf{c}}_{3 \mathrm{~b} \uparrow}^{+} \hat{\mathbf{c}}_{5 f \downarrow}^{+} \hat{\mathbf{c}}_{6 b \downarrow}^{+}|0\rangle$ in the four-electron system). The number of the basis vectors is calculated to be $C_{4 M}^{N_{e}}$, which gives the dimension of the Hamiltonian matrix ( $\mathrm{H}$ matrix). Diagonalization process of the $\mathrm{H}$ matrix can be simplified by reducing its dimension with the help of several symmetries in the system. Owing to the translational symmetry along the $\mathrm{x}(\mathrm{y})$ axis, the Hamiltonian conserves the total momentum $J_{\mathrm{x}}\left(J_{y_{N_{\mathrm{e}}}}\right.$ in the $\mathrm{x}$ (y) direction [17] [18] [19] [20]. Along the $y$ axis, for instance, $J_{\mathrm{y}}=\sum_{\mathrm{n}=1}^{N_{\mathrm{e}}} j_{\mathrm{n}}(\bmod M)$ is conservative, hence the $\mathrm{H}$ matrix as well as the basis vectors $\stackrel{n=1}{c}$ be divided into $M$ independent blocks corresponding to $M$ different values of $J_{y}$, the dimension of the blocks are merely $1 / M$ of that of the original $\mathrm{H}$ matrix [17] [18]. Let the common factor of $N_{\mathrm{e}}$ and $M$ be $C$. Then, the $\mathrm{H}$ matrix can be divided into independent $M \times C$ blocks with different combination of $\left(J_{x}, J_{y}\right), J_{x}, J_{y} \in[0, C-1]$, correspondingly, and its di- 
mension is reduced to about $1 / \mathrm{MC}$. The wave vector in the system is defined by $\mathbf{k}=\left(k_{x}, k_{y}\right)=\left(J_{x} 2 \pi / a, J_{y} 2 \pi / b\right)$ [17] [18] [19]. Since the z-component of the total spin $\left(S_{\mathrm{z}}\right)$ is conserved in the system, each block above can be divided further into $N_{\mathrm{e}}+1$ independent blocks keeping $S_{\mathrm{z}}$ from $-N_{\mathrm{e}} / 2$ to $N_{\mathrm{e}} / 2$, respectively. In the ED calculations, we compute the matrix elements of these blocks and diagonalize them numerically. The aspect ratio $a / b$ is fixed at 1.0.

In this study, a finite size $v=2 / 3$ bilayer $\mathrm{QH}$ systems containing four electrons is chosen to execute the ED calculations. Henceforth, in the numerical results, the length and energy units are selected by $I_{B}$ and Coulomb energy scale $E_{\mathrm{C}}=e^{2} / 4 \pi \varepsilon l_{B}$, respectively.

\section{Numerical Results and Discussion}

The low-lying energy spectra of the $v=2 / 3$ bilayer QH system with the fixed $d=$ 1.0 for several values of $\Delta_{\mathrm{SAS}}$ and $\Delta_{\mathrm{Z}}$ are presented in Figure 1. The energies are plotted by different marks indicating different total spins $S_{z}$, and are measured from the ground-state energies indicated by arrows. Wave vectors $k$ are normalized as $k l_{\mathrm{B}}$, and $\left(J_{\mathrm{x}}, J_{\mathrm{y}}\right)$ combinations contained in $k$ are also represented. It

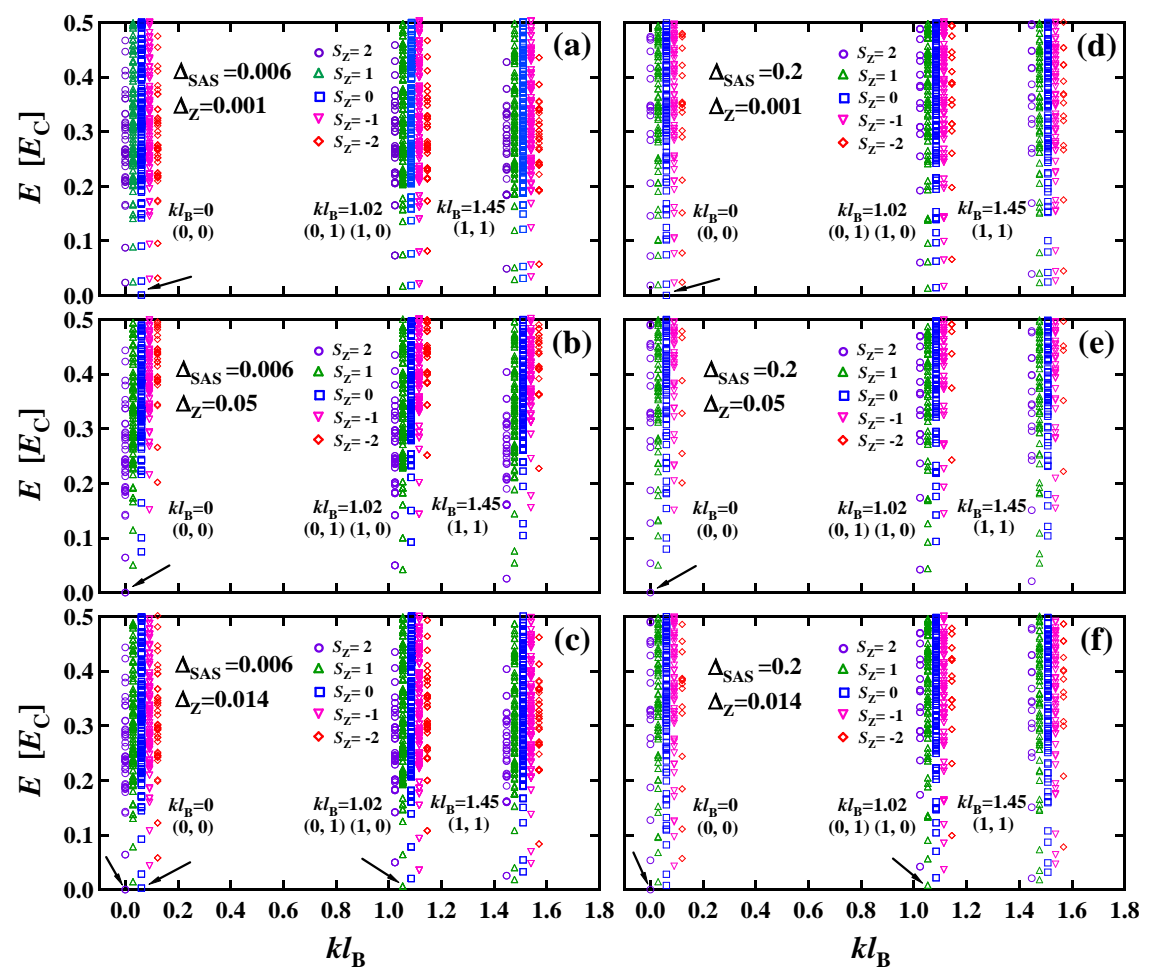

Figure 1. Energy spectrum of finite size $v=2 / 3$ bilayer $\mathrm{QH}$ systems (four electrons) for several values of $\Delta_{\mathrm{SAS}}$ and $\Delta_{\mathrm{Z}}$ at fixed $d=I_{\mathrm{B}}$. Eigenenergies are in unit of $\left(l_{\mathrm{B}}\right.$ is the magnetic length), and are plotted by different marks indicating different total spins $S_{z}$ (leaving space between the neighboring $S_{z}$ in the same $k$ for clarity). The wave number $k$, normalized as $k l_{\mathrm{B}}$, is shown together with $\left(J_{\mathrm{x}}, J_{\mathrm{y}}\right)$. The energies are measured from those of the ground states indicated by arrows. (a) $\Delta_{\mathrm{SAS}}=0.006, \Delta_{\mathrm{Z}}=0.001$. (b) $\Delta_{\mathrm{SAS}}=0.006, \Delta_{\mathrm{Z}}=$ 0.05. (c) $\Delta_{\mathrm{SAS}}=0.2, \Delta_{\mathrm{Z}}=0.014$. (d) $\Delta_{\mathrm{SAS}}=0.2, \Delta_{\mathrm{Z}}=0.001$. (e) $\Delta_{\mathrm{SAS}}=0.2, \Delta_{\mathrm{Z}}=0.05$. (f) $\Delta_{\mathrm{SAS}}$ $=0.2, \Delta_{\mathrm{Z}}=0.014$. 
should be noted that $k=1.02 / I_{\mathrm{B}}$ states are two-fold degenerate because $(0,1)$ and $(1,0)$ are equivalent. Figures $1(a)-(c)$ represent the spectra at small $\Delta_{\mathrm{SAS}}=0.006$. Actually, in these figures the marks of the ground state are almost doubly degenerate, composed of symmetry and antisymmetry states. While from the spectra at relatively large $\Delta_{\mathrm{SAS}}=0.2$ in Figures $1(\mathrm{~d})$-(f), we find that all the ground states become non-degenerate states. On the other hand, the spin polarizations of the ground states are determined by $\Delta_{\mathrm{Z}}$. As $\Delta_{\mathrm{Z}}$ is equal to a small value of 0.001 in Figure 1(a) and Figure 1(d), the ground states have the total spin $S_{z}=0$, being the SU states. When $\Delta_{\mathrm{Z}}$ becomes as large as 0.05 in Figure $1(\mathrm{~b})$ and (e), the ground states turn out to be the SP states with $S z=2$. The ground states have $k=$ 0 , as implies that they are homogeneous states. Both SP and SU ground states are $\mathrm{QH}$ states, where the lowest excited states have nonzero energy gap: They compose SP and SU phases. When $\Delta_{\mathrm{Z}}$ has an intermediate value of 0.014 as shown in Figure 1(c) and Figure 1(f), there are several states with almost equivalent lowest gap energies but different $S_{z}$, thus they all may become the ground states in these crossover regions when $\Delta_{Z}$ is slightly changed.

We introduce the most important basis states (MIBSs) of the ground state to investigate the properties of them. We find that two degenerate ground states in Figure 1(b) have the same representative basis vectors in the MIBSs. They are $\{2 \mathrm{f} \uparrow, 3 \mathrm{f} \uparrow, 5 \mathrm{f} \uparrow, 6 \mathrm{f} \uparrow\},\{2 \mathrm{~b} \uparrow, 3 \mathrm{~b} \uparrow, 5 \mathrm{~b} \uparrow, 6 \mathrm{~b} \uparrow\},\{1 \mathrm{f} \uparrow, 2 \mathrm{f} \uparrow, 3 \mathrm{f} \uparrow, 4 \mathrm{f} \uparrow\}$ and $\{1 \mathrm{~b}$ $\uparrow, 2 \mathrm{~b} \uparrow, 3 \mathrm{~b} \uparrow, 4 \mathrm{~b} \uparrow\}$, with the amplitudes of $(0.6279,0.6269,0.3250,0.3245)$ and $(0.6269,-0.6279,0.3245,-0.3250)$ for two degenerate ground states, respectively. Although the absolute value of the amplitudes are almost equal, the signs of the second and the fourth components are opposite, implying that they represent the symmetry and antisymmetry states, respectively. This phenomenon generally appears in the other degenerate ground states when $\Delta_{\mathrm{SAS}}$ is small.

Figure 2 demonstrates the energy gaps $E_{\text {gap }}$ between the lowest two eigenstates as a function of $\Delta_{\mathrm{SAS}}$ for several values of $d$ in the $v=2 / 3$ bilayer QH system for $\Delta_{\mathrm{Z}}=0.001$ and 0.05 . The lowest two eigenstates are the symmetric and antisymmetric states. As expected, they are almost degenerate in the small $\Delta_{\mathrm{SAS}}$ regions. With the increase of $\Delta_{\mathrm{SAS}}, E_{\text {gap }}$ smoothly widen, implying that the degeneracy of two states is resolved gradually, and finally tend to saturation points where the energies of the antisymmetric states exceed those of excited states created on the symmetric states. On the other hand, we notice that, when $d$ changes from 0 to 10 , the degenerate regions expand gradually and get to the limits. It is probably because the interlayer Coulomb energies exponentially decrease when $d$ increases, while the intralayer Coulomb energies are independent from $d$. It is conjectured that the large difference between the intralayer and interlayer Coulomb energies will increase the degeneracy of the ground states. The general features in Figure 2(a) and Figure 2(b) are similar, while the only difference between them is the values of $E_{\text {gap }}$ at the saturation points at large $\Delta_{\mathrm{SAS}}$, resulting from different excited states.

We plot the image of $E_{\text {gap }}$ in the $\Delta_{\mathrm{SAS}-\Delta \mathrm{Z}}$ plane with the choice of $d=1.0$ in the 


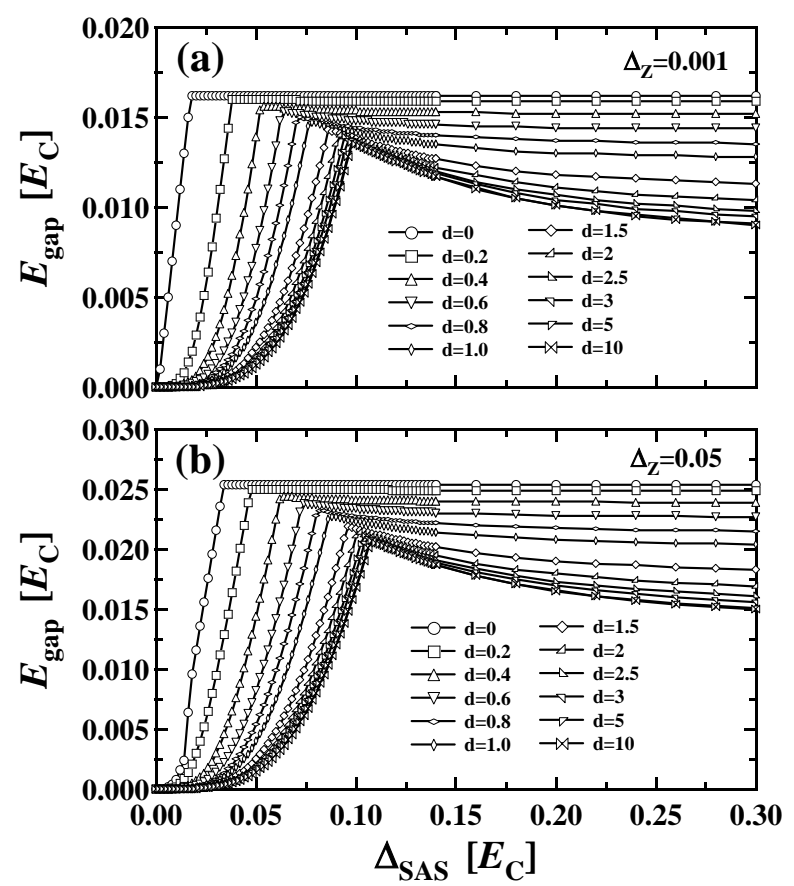

Figure 2. Energy gaps Egap between the lowest two eigenstates as a function of $\Delta_{\mathrm{SAS}}$ for several values of $\mathrm{d}$ in finite size $v=2 / 3$ bilayer QH systems (a) When $\Delta_{\mathrm{Z}}=0.001$; (b) When $\Delta_{\mathrm{Z}}=0.05$. The energy and $\mathrm{d}$ are in units of $E_{\mathrm{C}}=e^{2} / 4 \pi \varepsilon l_{B}$ and $I_{\mathrm{B}}$, respectively.

right side of the vertical dotted line in Figure 3(a), where $E_{\text {gap }}$ represents the energy gap between the lowest first and second eigenstates for the non-degenerate ground states case. We also plot the image of $E_{\text {gapD }}$ in the left side of the vertical dotted line in Figure 3(b), where $E_{\text {gapD }}$ represents the energy gap between the second and third eigenstates for the degenerate ground states case. The SP and SU phases occupy the high- and low- $\Delta_{\mathrm{Z}}$ regions with relative large $E_{\text {gap }}\left(E_{\text {gap }}\right)$ respectively. Because the phase transition between them is continuous in the finite size systems, in this paper, the crossover (CR) region is defined by the remaining part around the phase transition boundary where the values of $E_{\text {gap }}\left(E_{\text {gap }} \mathrm{D}\right)$ are less than one tenth of the average values of the $E_{\text {gap }}$ $\left(E_{\text {gap }}\right)$ in the SP and SU phases. The solid lines in the Figure 3(a) and Figure 3(b) give the upper and lower limits of the CR regions, and the dashed lines between them indicate the spin-flipping positions (between $S_{\mathrm{z}}=0$ and $S_{\mathrm{z}}=2$ ). The points A-E plotted in the figure are the experimental results of the phase boundaries (from N. Kumadaet al, Y. D. Zhenget al). The most significant feature in Figure 3(a) and Figure 3(b) is that, the SP-SU phase transition region (or the CR region) is limited in a narrow areawith $\Delta_{Z}$ from 0.009 to 0.014 . Though the transition region slightly bends toward the low $\Delta_{\mathrm{Z}}$ side when $\Delta_{\mathrm{SAS}}$ increases, we can say generally it is only weakly dependent on $\Delta_{\mathrm{SAs}}$. This fact gives the reliable evidence that the SP-SU phase transitions are determined by the competition between $\Delta_{\mathrm{Z}}$ and $\Delta_{\mathrm{C}}$. It should be emphasized that in the $v=2 / 3$ system, because the electrons only occupy the symmetric level, the factor $\Delta_{\mathrm{SAS}}$ representing the 

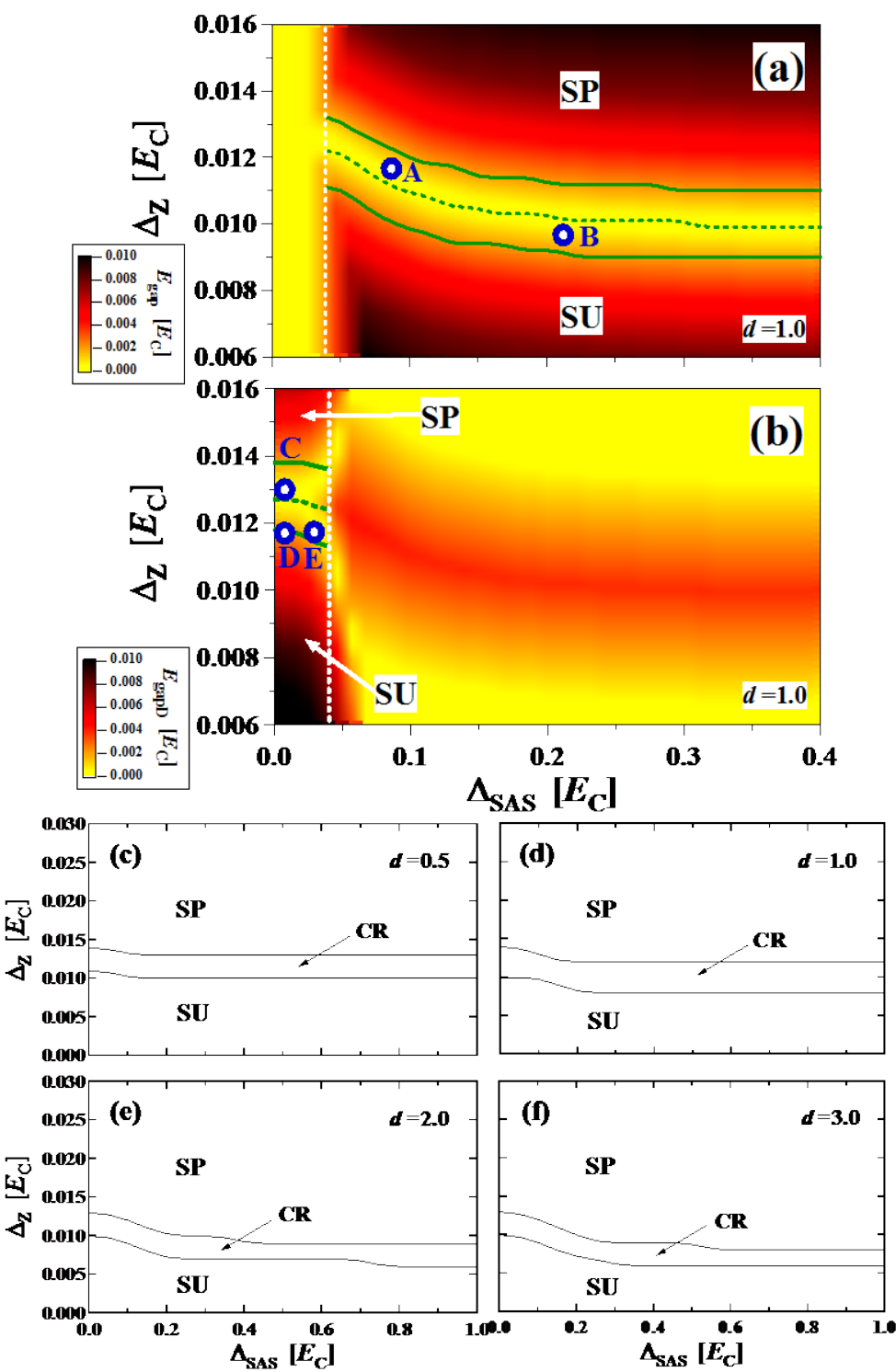

Figure 3. Image plot of energy gaps $E_{\text {gap }}$ between the lowest first and second eigenstates (a) and $E_{\mathrm{gapD}}$ between second and third eigenstates (b) in the $\Delta_{\mathrm{SAS}} \Delta_{\mathrm{Z}}$ plane in finite size $v$ $=2 / 3$ bilayer $\mathrm{QH}$ systems. Symbols SP, SU and CR denote the spin polarized, unpolarized phases and the crossover region, respectively. The solid lines give the upper and lower limits of the CR regions, and the dashed lines between them indicate the spin-flipping positions. The points A-E are the experimental results of the phase boundaries in the $v=$ $2 / 3$ bilayer QH systems; (c)-(f) SP, SU and CR regions in the $\Delta_{\mathrm{SAS}} \Delta_{\mathrm{Z}}$ planes for several values of d: (c) $d=0.5$; (d) $d=1.0$; (e) $d=2.0$; (f) $d=3.0$. The energy and $d$ are in units of $E_{\mathrm{C}}=e^{2} / 4 \pi \varepsilon l_{B}$ and $l_{\mathrm{B}}$, respectively.

energy gap between the symmetric and antisymmetric levels, will not affect the phase transition boundary. For this reason, the $\Delta_{\mathrm{Z}}$ holds a nonzero value in the transition region, even if $\Delta_{\mathrm{SAS}}$ vanishes. Figures $3(\mathrm{c})$-(f) present the SP, SU phases and CR region in the $\Delta_{\mathrm{SAS}}-\Delta_{\mathrm{Z}}$ plane for several values of $d$. When $d$ changes from 0.5 to 3.0 , the whole CR region slightly shifts to the low $\Delta_{\mathrm{Z}}$ side, and arrives to a limit about 0.005 . Qualitatively, $d$ has no great influence on the 
phase transition region.

\section{Analytic Investigation Using an Exactly Solvable Model}

The degeneracy of the ground states when $\Delta_{\mathrm{SAS}}$ is small can be investigated analytically by a two-electron model in the SP phase. With the help of the symmetries mentioned previously, the $\mathrm{H}$ matrix of this model can be divided into nine independent blocks. We write down five basis vectors corresponding to the block with the $J_{\mathrm{y}}\left(J_{\mathrm{x}}\right)$ value of 0 and the $S_{\mathrm{z}}$ value of 1 as $\varphi_{1}=\hat{\mathbf{c}}_{1 \mathrm{f} \uparrow}^{+} \hat{\mathbf{c}}_{2 \mathrm{f} \uparrow}^{+}|0\rangle$, $\varphi_{2}=\hat{\mathbf{c}}_{1 \mathrm{f}}^{+} \hat{\mathbf{c}}_{2 \mathrm{~b} \uparrow}^{+}|0\rangle, \quad \varphi_{3}=\hat{\mathbf{c}}_{1 \mathrm{~b} \uparrow}^{+} \hat{\mathbf{c}}_{2 \mathrm{f} \uparrow}^{+}|0\rangle, \quad \varphi_{4}=\hat{\mathbf{c}}_{1 \mathrm{~b} \uparrow}^{+} \hat{\mathbf{c}}_{2 \mathrm{~b} \uparrow}^{+}|0\rangle \quad$ and $\quad \varphi_{5}=\hat{\mathbf{c}}_{3 \mathrm{f} \uparrow}^{+} \hat{\mathbf{c}}_{3 \mathrm{~b} \uparrow}^{+}|0\rangle$, which belong to the SP phase. Using the Hamiltonian in Equation (1), we obtain a $5 \times 5$ block matrix calculated by the basis vectors above as follows:

$$
\left[H_{001}\right]=
$$

$$
\left(\begin{array}{ccccc}
A_{1221}-A_{1212}-\Delta_{\mathrm{Z}} & -(1 / 2) \Delta_{\mathrm{SAS}} & -(1 / 2) \Delta_{\mathrm{SAS}} & 0 & 0 \\
-(1 / 2) \Delta_{\mathrm{SAS}} & B_{1221}-\Delta_{\mathrm{Z}} & -B_{1212} & -(1 / 2) \Delta_{\mathrm{SAS}} & B_{2133} \\
-(1 / 2) \Delta_{\mathrm{SAS}} & -B_{1212} & B_{1221}-\Delta_{\mathrm{Z}} & -(1 / 2) \Delta_{\mathrm{SAS}} & -B_{1233} \\
0 & -(1 / 2) \Delta_{\mathrm{SAS}} & -(1 / 2) \Delta_{\mathrm{SAS}} & A_{1221}-A_{1212}-\Delta_{\mathrm{Z}} & 0 \\
0 & B_{2133}^{*} & -B_{1233}^{*} & 0 & B_{3333}-\Delta_{\mathrm{Z}}
\end{array}\right)
$$

where $A_{\mathrm{pqrs}}\left(\left\langle\phi_{p \mathrm{f}}, \phi_{q \mathrm{f}}|V| \phi_{r \mathrm{f}}, \phi_{\mathrm{sf}}\right\rangle \quad\right.$ or $\left.\left\langle\phi_{p \mathrm{~b}}, \phi_{q \mathrm{~b}}|V| \phi_{r \mathrm{~b}}, \phi_{\mathrm{sb}}\right\rangle\right)$ and $B_{\mathrm{pqrs}}(=$ $\left\langle\phi_{p \mathrm{f}}, \phi_{q \mathrm{~b}}|V| \phi_{\mathrm{rb}}, \phi_{\mathrm{sf}}\right\rangle$ or $\left\langle\phi_{p \mathrm{~b}}, \phi_{q \mathrm{f}}|V| \phi_{r \mathrm{f}}, \phi_{\mathrm{sb}}\right\rangle$ represent the intralayer and interlayer Coulomb interaction energies, respectively. The subscripts $\mathrm{p}, \mathrm{q}, \mathrm{r}$ and $\mathrm{s}$ denote the momentum index in the y direction. $\phi_{j \mu}$ is the single-electron wave function in the LLL. $A_{1221}$, for instance, is the direct Coulomb energy between two electrons in sites 1 and 2, while $A_{1212}$ is the exchange energy between them.

The block $\left[H_{001}\right]$ can be diagonalized analytically through the conventional method, and we derive all eigenvalues and eigenstates of the matrix characteristic equation $\left[H_{001}\right]\left(\begin{array}{lllll}A_{\varphi_{1}} & A_{\varphi_{2}} & A_{\varphi_{3}} & A_{\varphi_{4}} & A_{\varphi_{5}}\end{array}\right)^{\mathrm{T}}=E\left(\begin{array}{llllll}A_{\varphi_{1}} & A_{\varphi_{2}} & A_{\varphi_{3}} & A_{\varphi_{4}} & A_{\varphi_{5}}\end{array}\right)^{\mathrm{T}}$. The five exact eigenvalues $\left(E_{1}-E_{5}\right)$ of the block $\left[H_{001}\right]$ are given by

$$
\begin{gathered}
E_{1}=\frac{\left(A_{1221}-A_{1212}+B_{1221}-B_{1212}\right)-\sqrt{\Delta_{1}}}{2}-\Delta_{Z}, \quad E_{2}=A_{1221}-A_{1212}-\Delta_{Z}, \\
E_{3}=\frac{\left(A_{1221}-A_{1212}+B_{1221}-B_{1212}\right)+\sqrt{\Delta_{1}}}{2}-\Delta_{Z}, \\
E_{4}=\frac{\left(B_{3333}+B_{1221}+B_{1212}\right)-\sqrt{\Delta_{2}}}{2}-\Delta_{Z}, \\
E_{5}=\frac{\left(B_{3333}+B_{1221}+B_{1212}\right)+\sqrt{\Delta_{2}}}{2}-\Delta_{Z},
\end{gathered}
$$

with

$$
\begin{gathered}
\Delta_{1}=\left(A_{1221}-A_{1212}-B_{1221}+B_{1212}\right)^{2}+4 \Delta_{S A S}^{2}, \\
\Delta_{2}=\left(B_{3333}-B_{1221}-B_{1212}\right)^{2}+8\left|B_{1233}\right|^{2} .
\end{gathered}
$$

Since the relationship $A_{1221}-A_{1212}<B_{1221}-B_{1212}$ holds always in the system, one obtains provided $\Delta_{\mathrm{SAS}}=0$, exhibiting two degenerate ground states with 
equivalent eigenvalues $E_{1}$ and $E_{2}$. The eigenstates in this case are simply $\hat{\mathbf{c}}_{1 \mathrm{f}}^{+} \hat{\mathbf{c}}_{2 \mathrm{f} \uparrow}^{+}|0\rangle$ or $\hat{\mathbf{c}}_{1 \mathrm{~b} \uparrow}^{+} \hat{\mathbf{c}}_{2 \mathrm{~b} \uparrow}^{+}|0\rangle$ implying that electrons are restricted within one of the layers.

When $\Delta_{\mathrm{SAS}}$ departures from zero, $E_{1}$ is also separated from $E_{2}$ taking a lower value. Thus, the degeneracy of the ground states is solved gradually. However, when $\Delta_{\mathrm{SAS}}$ is sufficiently small, $E_{1}$ and $E_{2}$ are almost equal, and can be regarded as degenerate. In this case, the difference between $E_{1}$ and $E_{2}$ can be approximated by

$$
\left|E_{1}-E_{2}\right|=\Delta_{S A S}\left|\frac{\Delta_{S A S}}{A_{1221}-A_{1212}-B_{1221}+B_{1212}}\right| .
$$

It is obvious that the degeneracy of the ground states is increased not only by the small $\Delta_{\mathrm{SAS}}$ but also by the large difference between the intralayer and interlayer Coulomb energies, because $\left|E_{1}-E_{2}\right|$ is in proportion to the product of $\Delta_{\mathrm{SAS}}$ and the ratio of $\Delta_{\mathrm{SAS}}$ and $\left|\left(A_{1221}-A_{1212}\right)-\left(B_{1221}-B_{1212}\right)\right|$. Equation (5) reliably gives an analytic interpretation of the numerical results presented Figure 2. On the other hand, the eigenstates corresponding to $E_{1}$ and $E_{2}$ are expressed by

$$
\begin{aligned}
& \Psi_{E_{1}}=a\left(\hat{\mathbf{c}}_{1 \mathrm{f} \uparrow}^{+} \hat{\mathbf{c}}_{2 \mathrm{f} \uparrow}^{+}|0\rangle+\hat{\mathbf{c}}_{1 \mathrm{~b} \uparrow}^{+} \hat{\mathbf{c}}_{2 \mathrm{~b} \uparrow}^{+}|0\rangle\right)+b_{1} \hat{\mathbf{c}}_{\mathrm{ff}}^{+} \hat{\mathbf{c}}_{2 \mathrm{~b} \uparrow}^{+}|0\rangle+b_{2} \hat{\mathbf{c}}_{1 \mathrm{~b} \uparrow}^{+} \hat{\mathbf{c}}_{2 \mathrm{f} \uparrow}^{+}|0\rangle+b_{3} \hat{\mathbf{c}}_{3 \mathrm{f} \uparrow}^{+} \hat{\mathbf{c}}_{3 \mathrm{~b} \uparrow}^{+}|0\rangle, \\
& \Psi_{E_{2}}=(\sqrt{2} / 2)\left(\hat{\mathbf{c}}_{1 \mathrm{f}}^{+} \hat{\mathbf{c}}_{2 \mathrm{f} \uparrow}^{+}|0\rangle-\hat{\mathbf{c}}_{1 \mathrm{~b} \uparrow}^{+} \hat{\mathbf{c}}_{2 \mathrm{~b} \uparrow}^{+}|0\rangle\right) .
\end{aligned}
$$

For sufficiently small $\Delta_{\mathrm{SAS}}$, since coefficients $b_{1}-b_{3}$ are all close to zero, the two degenerate ground states with energies $E_{1}$ and $E_{2}$ are symmetric and antisymmetric states, as argued previously.

\section{Conclusion}

In summary, we have studied a typical bilayer FQH system with finite size using the numerical and analytic methods and provided evidential quantitative results. We have found some basic features of the ground states at the layer balanced point in the $v=2 / 3$ bilayer $\mathrm{QH}$ systems taking advantage of the ED calculations and the analysis of an exactly solvable two-electron system. When $\Delta_{\mathrm{SAS}}$ is small, the degeneracy of the ground states occurs depending on the relative strength of the intralayer and interlayer Coulomb energies. SP-SU phase transitions are continuous in the finite size systems, and are determined by the competition between $\Delta_{\mathrm{Z}}$ and Coulomb energy $\Delta_{\mathrm{C}}$, almost not affected by $\Delta_{\mathrm{SAS}}$. These features exhibit the essential difference between the $v=2 / 3$ bilayer FQH systems and the $v=2$ bilayer IQH systems, and the peculiar characteristics generally existing in most bilayer FQH systems. The ED numerical method and the exact-solution method employed in this paper also can be considered to be valuable in studies of other bilayer FQH systems.

\section{Acknowledgements}

This research was supported in part by Grants-in-Aid for the basic research and development of Mitsubishi Electric (China) Company Limited. 


\section{References}

[1] Klitzing, K.V., Dorda, G. and Pepper, M. (1980) Phys. Rev. Lett., 45, 494. https://doi.org/10.1103/PhysRevLett.45.494

[2] Tsui, D.C., Stormer, H.L. and Gossard, A.C. (1982) Phys. Rev. Lett., 48, 1559. https://doi.org/10.1103/PhysRevLett.48.1559

[3] Laughlin, R.B. (1983) Phys. Rev. Lett., 50, 1395. https://doi.org/10.1103/PhysRevLett.50.1395

[4] Halperin, B.I. (1984) Phys. Rev. Lett., 52, 1583. https://doi.org/10.1103/PhysRevLett.52.1583

[5] Jain, J.K. (1989) Phys. Rev. Lett., 63, 199. https://doi.org/10.1103/PhysRevLett.63.199

[6] Das Sarma, S. and Pinczuk, A. (editor) (1997) Perspectives in Quantum Hall Effects. Wiley, New York.

[7] Girvin, S.M. (1999) arXiv: cond-mat/9907002v1

[8] Zheng, L., Radtke, R.J. and Das Sarma, S. (1997) Phys. Rev. Lett., 78, 2453. https://doi.org/10.1103/PhysRevLett.78.2453

[9] Das Sarma, S., Sachdev, S. and Zheng, L. (1998) Phys. Rev. B, 58, 4672. https://doi.org/10.1103/PhysRevB.58.4672

[10] MacDonald, A.H., Rajaraman, R. and Jungwirth, T. (1999) Phys. Rev. B, 60, 8817. https://doi.org/10.1103/PhysRevB.60.8817

[11] Brey, L., Demler, E. and Das Sarma, S. (1999) Phys. Rev. Lett., 83, 168. https://doi.org/10.1103/PhysRevLett.83.168

[12] Burkov, A.A. and MacDonald, A.H. (2002) Phys. Rev. B, 66, Article ID: 115323.

[13] Lopatnikova, A., Simon, S.H. and Demler, E. (2004) Phys. Rev. B, 70, Article ID: 115325.

[14] Demler, E. and Das Sarma, S. (1999) Phys. Rev. Lett., 82, 3895. https://doi.org/10.1103/PhysRevLett.82.3895

[15] Yang, K. (1999) Phys. Rev. B, 60, 15578. https://doi.org/10.1103/PhysRevB.60.15578

[16] Schliemann, J. and MacDonald, A.H. (2000) Phys. Rev. Lett., 84, 4437. https://doi.org/10.1103/PhysRevLett.84.4437

[17] Yoshioka, D., Halperin, B.I. and Lee, P.A. (1983) Phys. Rev. Lett., 50, 1219. https://doi.org/10.1103/PhysRevLett.50.1219

[18] Yoshioka, D. (1984) Phys. Rev. B, 29, 6833. https://doi.org/10.1103/PhysRevB.29.6833

[19] Haldane, F.D.M. (1985) Phys. Rev. Lett., 55, 2095. https://doi.org/10.1103/PhysRevLett.55.2095

[20] Haldane, F.D.M. and Rezayi, E.H. (1985) Phys. Rev. Lett., 54, 237. https://doi.org/10.1103/PhysRevLett.54.237 